\title{
A Novel Network Resilience Predicting Method Based on Machine Learning
}

\author{
Weiqiang $\mathrm{Wu}^{2,3,4, a, *}$, Qilin Hou ${ }^{1,3, b}$, Lili Zhong ${ }^{1,3, ~ c}$, Hongbo Yan ${ }^{2, d}$, \\ Yiqiang Wan ${ }^{3, e}$, Jianglei Wang ${ }^{1, f}$, Jun $\mathrm{Li}^{1,9}$, Feifei He $\mathrm{H}^{1, \mathrm{~h}}$ \\ ${ }^{1}$ Technology Division, China Huarong Asset Management Co, Ltd. Beijing 100033, China. \\ ${ }^{2}$ Postdoctoral Workstation, China Huarong Asset Management Co, Ltd. Beijing 100033, China. \\ ${ }^{3}$ Huarong Rongtong (Beijing) Technology Co, Ltd. Beijing 100033, China. \\ ${ }^{4}$ School of Economics and Management, University of Chinese Academy of Sciences. Beijing \\ 100190, China \\ *, awuweiqiang@chamc.com.cn, bcharlie@chamc.com.cn, cllzhong@chamc.com.cn, \\ dyanhongbo@chamc.com.cn, ewanyiqiang@chamc.com.cn, fwangjianglei@chamc.com.cn, \\ glijunh@chamc.com.cn, hhefeifei@chamc.com.cn
}

\begin{abstract}
Network resilience is an important indicator for measuring the pros and cons of faulttolerant strategies. For the resilience prediction of the network design stage, the traditional prediction methods can only be modeled and forecasted based on fault tolerance strategies based on a single network, and the impact of fault tolerant strategies deploying on multi-layer of network cannot be considered. For solving this problem, this paper proposed a network resilience prediction method based on network resilience theory and machine learning. First, a quantitative model of network fault tolerance strategy is established, and based on this model, the modeling method of the protocol stack is improved on the NS3 platform for simulation implement. A large number of structured data that can characterize network resilience are generated through simulation experiments. Then, a network resilience prediction model of single hidden layer feed-forward neural network (SLFN) is established based on the Extreme Learning Machine (ELM) theory. This model can better solve the overfit problem of traditional ELM. Finally, the prediction model was verified according to comparing with the traditional model-driven resilience prediction method. This method not only has a higher prediction accuracy, but also solves the problem that the traditional method cannot model the coupling relationship between fault-tolerant strategies, and thus cannot be based on the hybrid faulttolerant strategy for resilience prediction. The case verification shows that this method can effectively predict the network resilience considering fault-tolerant strategies. Through cross-validation, the accuracy rate is more than $96 \%$, which helps to consider the development of fault-tolerant design of hybrid fault-tolerance strategies.
\end{abstract}

Keywords: Network Resilience, Fault Tolerance, Machine Learning, ELM.

\section{Introduction}

The network fault-tolerant strategy can make the network resist the fault through the active or passive fault-tolerance mechanism when it suffers from failure, and guarantee the reliability of the network [1], [2]. In order to ensure the best fault tolerance capability of the system, multiple fault tolerance mechanisms are usually deployed at different network layers according to the layered features of the network to form a hybrid fault tolerance strategy to improve the fault tolerance of the network, thereby ensuring network reliability [4]. The assessment of network fault tolerance is currently achieved through qualitative and quantitative assessment of network resiliency [5] Network resiliency is defined as the ability of a network to recover to its normal state after an attack or failure. Therefore, considering the fault-tolerant strategy, assessing and predicting the resilience of the network is an important issue in the network design phase.

In order to assess and predict the elasticity of the network, relevant scholars have conducted a lot of research. Most of the research focuses on network elasticity assessment. The research on the evaluation of network elasticity is mainly based on the elastic evaluation of the model. According to S. Hosseini and other scholars [6], the methods of network resilience assessment are classified. 
According to different fault tolerance mechanisms, resilience assessment can be divided into two types based on structure and topology. Among them, based on the structural and topological resilience assessment, the scholars mainly modeled the robustness and tolerance of the network physical layer topology, and then analyzed the network resilience based on the model. For example, scholars such as A. Sydney [7] proposed a measure of the resilience of the network structure through the natural topology of the network topology based on complex network theory; further, MJF Alenazi et al. [8] proposed using the network "spectrum measure" to measure the network resilience of structure, and at the same time it proves that the spectral measure is superior to other connectivity parameters and can better represent the resilience of the network structure. The research of resilience assessment based on network performance is mainly based on the model of network state, such as time delay and packet loss rate. Researchers such as JPG Sterbenz [5] used the state-space method to model the network operating state and evaluated the network resilience by the level of network recovery after the attack. Further, D. Zhang et al. [9] considering the impact of mobility on network resilience, JP Rohrer et al. [10] proposed that network resilience is the result of the joint impact of physical layer topology fault tolerance and logical layer fault tolerance protocol. The directly prediction of network resilience is an emerging hot spot in the field. The main idea is still based on network operating parameters and design parameters, and modeling of network resilience takes these parameters and the level of network elasticity. Mapping relationships to predict network resiliency [11]-[15]. At present, there are more representative researches: J. Gao and A.-L. Barabási [13] propose a prediction method based on "patterns": This method is based on complex network theory and abstracts the network control related structural parameters and network status. The mapping of parameters abstracts the multi-dimensional dynamic model of the network into a one-dimensional model to form a model related to elasticity, and then predicts the network resilience through the network model.

In summary, most current network resilience prediction researches are expansion of resilience assessment. The methods based on these researches are used to predict network elasticity according to existing network structure and other design parameters. However, as a complex object, the network presents the characteristics of dynamic and coupling. Therefore, there is such a coupling and influence relationship within the hybrid fault-tolerance strategy. Existing resilience prediction methods can only predict a single fault-tolerant strategy. For example, in a fault-tolerant topology, network resilience can be predicted through structural parameters of a fault-tolerant topology, and resilience is based on logical layers or multi-layer hybrid fault-tolerance strategies cannot be predicted through the same approach. Due to the lack of quantitative analysis and modeling research of fault-tolerant strategies at the logical layer, traditional elasticity prediction methods cannot support the consideration of resilience prediction under the coupling of multiple fault-tolerance mechanisms.

For solving the mentioned problems, this paper proposed a data-driven resilience prediction method based on a combination of machine learning and resilience theory. Based on the quantitative modeling of network fault tolerance strategies, the advantages of machine learning methods in the field of nonlinear system pattern recognition and linear fitting [16] are adopted, and the emerging high-efficiency machine learning algorithm-ELM is adopted [17] as well for predicting of network resilience. The proposed approach not only fully considers the influence of fault-tolerance strategies at different layers of the resilience of the entire network, but also can intuitively reflect the trend of resilience changes of networks under different fault-tolerance strategies. The theory fruit can support the study of the coupling of hybrid fault-tolerance strategies on system reliability.

\section{Network Resilience Modeling}

According to the definition of the network layering theory, the applications of the network and the functions they has are implemented based on the transmission protocols of the network physical layer and logical layer routing. The routing protocol as a rule that specifies the path of data transmission on the network directly affects the normal operation of network functions and applications. The routing table generated by the routing protocol is based on the underlying physical layer connectivity path. On the macro time scale, the physical layer of the network, and the logical layers generated by 
logic rules such as routing protocols above the physical layer, each exhibit temporal and spatial dynamic characteristics over time in the structural and spatial dimensions. [14] Based on this, in order to describe the spatio-temporal dynamic characteristics of this kind of network feature, this paper proposed a space-time dynamic network model.

\subsection{Network Time-Spatial Feature}

Applications and functions are operated in the routes according to routing protocols. The spatial feature of routes is varying with the time process as the Fig 1. Shown:

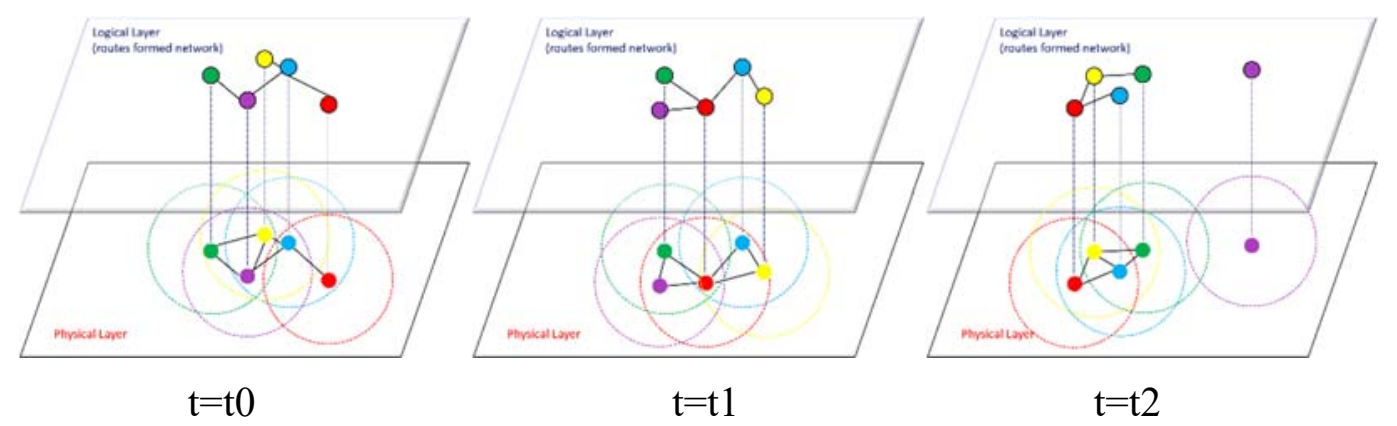

Fig. 1. Different Topology of Routes and Links in Different Time Profiles

Thus we can abstract the routes according to routing protocols as a network. Here we considered physical connective exhibiting on physical layer, and based on physical connective, the logical connective exhibiting on logical layer, dynamic features can be observed in time and spatial aspects: due to nodes' movement features, the physical links on physical layer and routes on logical layer are not fixed, meanwhile, the fault tolerance mechanism against failures is another factor which can affect network spatial structure. Therefore, the different routing protocols which have different rout discovery and maintenance mechanisms will also result in the different structure behaviors of the routes abstracted networks. The structure spatial dynamic process is expressing in time dimension.

So we considered the abstracted network formed by routing protocol possessing time-spatial features, and proposed "Time-Spatial" network model. We will use the Time-Spatial network model to describe time and spatial variation which is caused by routing protocols and fault tolerance principle, and carry on the resilience measurement on the network model as well.

\subsection{Time-Spatial Network Model Definition}

Routes discovery and maintenance according to routing protocols depend on the connection of physical layer, so the upper Time-Spatial network model also takes into account the physical network model. Meanwhile, the connectivity of MANET physical layer depends on the distance between nodes and the radius of signal transmission. About this property of physical layer, there are several random graph models are properly to model the physical layer of MANET, such as Gilbert graphs, Waxman graphs and Gabriel graphs [9], etc. With the purpose of closing the real usage profile and comparison, in this paper, we preferred Waxman graph as the physical layer model and our proposed Time-Spatial network model is above the physical layer as the logical layer.

Definition 1. Physical layer network model: The construction of physical layer network should yield Waxman model:

$$
P(u, v)=\beta e^{\frac{-d(u, v)}{L \alpha}}
$$

Where $\beta, \alpha \in(0,1]$, and $\mathrm{L}$ is the maximum distance between any two nodes, $\beta$ denotes the density of links, $\alpha$ corresponds to a high ratio of long links to short links.

Definition 2. Time-Spatial network model: Here we proposed the definition of Time-Spatial network model: MANET can be considered as a directed graph: $G=(V, E), \mathrm{V}$ is the set of the whole network, the number of the nodes in the set is $|V|$, for $u \in V,\langle u, v\rangle$ denotes valid path from $u$ to $\mathrm{v}$, denoted by: $e<u, v>,(u, v)$ express the link between $\mathrm{u}$ and $\mathrm{v}$, denoted by: $e(u, v)$. 
$E=\{e<u, v>\mid u \in V, v \in V\}$ is defined as valid edge. The number of edges or links is defined as $|E|$. Define the function $\mathrm{W}: E \rightarrow R+, R+$ is a subset of the positive real number, $W(e<u, v>)$ express the weight of edge: $e\langle u, v\rangle$, denoted by $w(u, v)$. If $w(u, v)$ is $+\infty$, then the link $e\langle u, v\rangle$ is break. The partially ordered set which is formed by $V:\left\{v_{1}, v_{2}, \ldots, v_{j-1}, v_{j}, v_{j+1}, \ldots, v_{n}\right\}$, is denoted a path which source node is $v_{1}$, destination node is $v_{n}$, and the node number of the path is $\mathrm{n}, \forall k(2 \leq k \leq n), v_{k-1}$ is the upstream node in the path of $v_{k} . \forall m(1 \leq m \leq n-1)$. The node $v_{m+1}$ is the downstream node in the path of node $v_{m}$. For $v_{i} \in v, v_{j} \in v, \operatorname{Path}\left(v_{i}, v_{j}\right)$ express the set of path that the source node is $v_{i}$, the destination node is $v_{j}$, in other words, it is the edges set of Time-Spatial Network. We assumed: $\operatorname{path}\left(v_{i}, v_{j}\right) \in \operatorname{Path}\left(v_{i}, v_{j}\right)$, then define the function: $f: \operatorname{Path}\left(v_{i}, v_{j}\right) \rightarrow \mathbb{R}+, f\left(\operatorname{path}\left(v_{i}, v_{j}\right)\right)$ denotes the evaluation function of path $\left(v_{i}, v_{j}\right)$.

The purpose of the routing that source node is $v_{i}$ and destination node is $v_{j}$, is to find out an $\operatorname{path}^{*}\left(v_{i}, v_{j}\right)$ which the evaluation function is optimal, satisfy the formula:

$$
\exists \operatorname{path}^{*}\left(v_{i}, v_{j}\right) \forall \operatorname{path}\left(v_{i}, v_{j}\right), f\left(\operatorname{path}^{*}\left(v_{i}, v_{j}\right)\right) \leq f\left(\operatorname{path}\left(v_{i}, v_{j}\right)\right)
$$

subject to:

$$
\operatorname{path}^{*}\left(v_{i}, v_{j}\right) \in \operatorname{Path}\left(v_{i}, v_{j}\right), \operatorname{path}\left(v_{i}, v_{j}\right) \in \operatorname{Path}\left(v_{i}, v_{j}\right)
$$

\subsection{Method of Network Fault Tolerance Modeling Based on Time-Spatial Model}

From network science perspective, the network structure or topology features can indicate certain of operation behaviors. Therefore, if we consider the resilience as a part of the network's behaviors, the network's structure will affect resilience features.

Besides the metrics mentioned in section 2.2, the special metrics for evaluating the network's robustness and resilience taking account to network structure metrics were also proposed, e.g. Spectral metrics, Nature connectivity, Algebraic connectivity[6],[13],[15], etc. Although all of them are fit for measuring the network structure robustness, it cannot completely reflect the impact from routing mechanism to the network directly. Recent years, some scholars are start to consider the packet transmission impacts and proposed Flow Robustness [14] metrics to measure the physical layer flow robustness (phyFR) as resilience assessment of network, ref.10 compared Flow Robustness with other structural based metrics, and it showed higher accuracy than others:

$$
\text { phyFR }_{=} \frac{\sum_{i=1}^{k} \mid \text { Component }_{i} \mid\left(\mid \text { Component }_{i} \mid-1\right)}{|n|(|n|-1)}
$$

Where let $G=(n, l)$ be the graph representing the given network. Let $\left\{\right.$ Component $\left._{i} ; 1<i<k\right\}$ be the set of components in graph G. the complexity for calculating the resilience by using phyFR is hard to find the components size in network topology graph. Hence, we may count edges' number instead of counting nodes number, because the number of links and edges are easy to collect during the simulation process that is considering routing mechanism.

Combined the metrics we introduced, here are steps of our measuring method:

Step 1. Based on the Time-Spatial Network Model, build a spatial profile of the network: (express as a matrix), and the profile can describe the connection status of the nodes in Time-Spatial Network at per time step: 


$$
A[i, j]= \begin{cases}w_{i j}, & <v_{i}, v_{j}>\in E(G) \\ 0, & <v_{i}, v_{j}>\notin E(G)\end{cases}
$$

Step2. Analyze the flow robustness of Time-Spatial (TSFR) network model.

$$
T S F R=\frac{\sum_{i=1}^{k}\left|C_{i}\right|\left(\left|C_{i}\right|-1\right)}{|n|(|n|-1)}, \quad 0 \leq T S F R \leq 1
$$

Where the definitions of parameters are follow eq. (4) introduced, but the analysis object is TimeSpatial network model. With the purpose of simplify the complexity, we can also count edges or links instead of counting $C_{i}$. As we known, the n nodes can construct $n(n-1) / 2$ edges, hence if where $e_{i}$ is the edge number of $C_{i}$, the eq. (4) can be transform to:

$$
\operatorname{TSFR}=\frac{\sum_{i=1}^{k} e_{i}}{E}
$$

Where $\mathrm{E}$ is the total number of possible network edges. Here we proposed a simple proof:

$$
e_{i}=\frac{\left|C_{i}\right|\left(\left|C_{i}\right|-1\right)}{2}=\frac{\left|C_{i}\right|^{2}-\left|C_{i}\right|}{2} \Rightarrow\left|C_{i}\right|^{2}-\left|C_{i}\right|-2 e_{i}=0
$$

Solving eq. (6):

$$
\begin{gathered}
\left|C_{i}\right|=\frac{1+\sqrt{1+8 e_{i}}}{2} \\
\because \frac{|n|(|n|-1)}{2}=E \\
\therefore|n|^{2}-|n|-2 E=0 \Rightarrow|n|=\frac{1+\sqrt{1+8 E}}{2}
\end{gathered}
$$

Substitute eq. (9) and eq. (11) to eq. (6), we can obtain eq. (7).

Step 3. Measuring the Resilience of Network: Refer to the resilience calculating method from Alenazi[10] et. al, we used Logical Fault Tolerance (LFT) to denote the network resilience: $\operatorname{STSFR}_{n}=\sum_{i=1}^{n} T S F R_{i}$.

\section{Network Resilience Prediction Model Based on ELM}

Based on the network fault tolerance model, this paper extracts the corresponding structured data from the network operating data according to the parameters defined by the proposed model, and uses the network resilience prediction model to predict the network resilience. Reference [13] mentioned that the traditional mathematical method of describing system resilience is to use a one-dimensional nonlinear dynamic equation to approximate the behavior of a complex system:

$$
\frac{\mathrm{d} x}{d t}=f(\beta, x)
$$


In eq. (12) $f(\beta, x)$ denotes the resilience of system, $\beta$ denotes the captured change of environment. Therefore, a network which was constructed by $\mathrm{N}$ components, the states set of nodes is $x=\left(x_{1}, \cdots, x_{N}\right)^{T}$, subject to the Coupled nonlinear equation:

$$
\frac{d x_{i}}{d t}=F\left(x_{i}\right)+\sum_{j=1}^{N} A_{i j} G\left(x_{i}, x_{j}\right)
$$

Nonlinear function $F\left(x_{i}\right)$ and $G\left(x_{i}, x_{j}\right)$ represents the dynamic rules of the control system components, the weight matrix $A_{i j}$ reflects the relationship between components. Function $F\left(x_{i}\right)$ and $G\left(x_{i}, x_{j}\right)$ can be selected to form eq. (13) for system modeling and obtain the resilience value. As an excellent approximation approach, machine learning would be an ideal choice for resilience prediction. Among them: combined with activation function and bias, $f\left(a_{i} \boldsymbol{x}+b_{i}\right)$ can be used to characterize the dynamic rules of the system reflected by data driven. It is Similar to $F\left(x_{i}\right)$ and $G\left(x_{i}, x_{j}\right)$ in equation (13), the trained output weight matrix $\hat{\boldsymbol{\beta}}$ can be used to represent $A_{i j}$.

Therefore, regarding the network resilience prediction model, this paper uses the ELM to construct the network elasticity prediction model. As a machine learning method that has become popular in recent years, the over-limit learning machine is a feedforward, single hidden layer neural network model. Compared with other machine learning methods, it has the advantages of high speed and generalization ability [17]. Therefore, in this paper, ELM was adopted as the basis for the resilience prediction model.

\subsection{The Definition of Prediction Model Based on ELM}

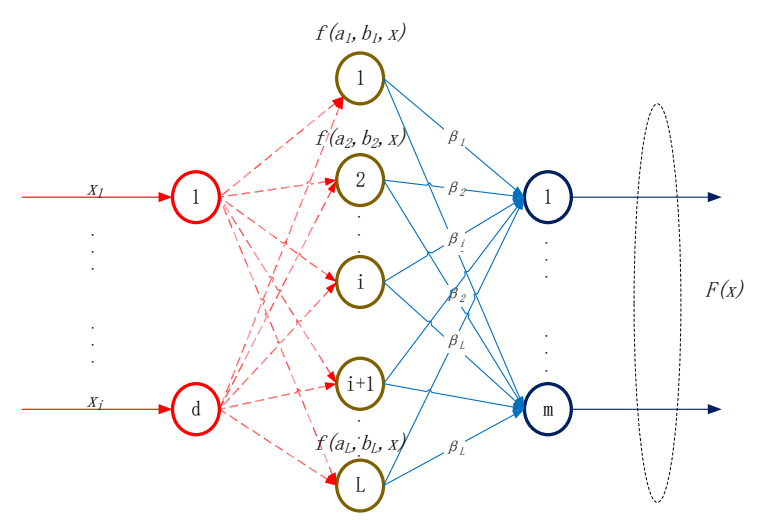

Fig. 2. ELM Structure

As shown in Fig. 2, a typical ELM model, $(a, b)$ is the input weight and bias (threshold) of the hidden node, and the training sample set is $(\mathrm{x}, \mathrm{T})$ (corresponding to a set of training samples. ) where: $\mathrm{x}$ represents the training sample's independent variables, that is, it can correspond to various faulttolerance models, various resilience-relevant parameters (phyFR, TSFR, etc.), and various network design or configuration parameters, such as topology, structure, mobile mode, traffic model, etc. $t$ represents the dependent variable, which can correspond to the actual level of network operation, that is, the parameter that can represent the network operating capability corresponding to the resilience value, such as the success rate of packet transmission, packet loss rate, and delay. The hidden layer mapping function (activation function) is denoted as $\mathrm{f}(\mathrm{x})$, and in order to ensure that the activation function is continuously differentiable, the sigmoid function is selected: $f(x)=\frac{1}{1+e^{-x}}$. The output weight is denoted as $\beta$, the number of nodes in the hidden layer is L, and Oi represents the learning error of the network. The neural network is expressed as a loss function: 


$$
O_{i}=\left\|\sum_{i=1}^{L} \beta_{i} f\left(a_{i} x+b_{i}\right)-t_{i}\right\|, \boldsymbol{x}=\left[x_{1}, x_{2}, \ldots, x_{L}\right]^{T}
$$

Set the output matrix of the hidden layer to $H$, the expected output to $\boldsymbol{T}$, then:

Therefore, we can obtain:

$$
\boldsymbol{H}\left(a_{1}, a_{2}, \ldots, a_{L}, b_{1}, b_{2}, \ldots, b_{L}, x_{1}, x_{2}, \ldots, x_{L}\right)=\left[\begin{array}{ccc}
f\left(a_{1} X_{1}+b_{1}\right) \cdots f\left(a_{L} X_{1}+b_{L}\right) \\
\vdots & \cdots & \vdots \\
f\left(a_{1} x_{L}+b_{1}\right) \cdots f\left(a_{L} x_{L}+b_{L}\right)
\end{array}\right]
$$

$$
\boldsymbol{O}=\|\boldsymbol{H} \boldsymbol{\beta}-\boldsymbol{T}\|
$$

among them: $\boldsymbol{\beta}=\left[\beta_{1}, \beta_{2}, \ldots, \beta_{L}\right]^{T} \quad \boldsymbol{T}=\left[t_{1}, t_{2}, \ldots, t_{L}\right]^{T}$

When the learning error is 0 , the learning machine is considered to have the best learning ability, then this time: $\boldsymbol{H} \boldsymbol{\beta}=\boldsymbol{T}$, where $\boldsymbol{H}$ is the known part (input weights, offsets, training sample variables), $\boldsymbol{T}$ is also for the known part (training sample dependent variable). Hence, the process of learning with an ELM is the process of obtaining $\boldsymbol{\beta}$ from $\boldsymbol{H} \boldsymbol{\beta}=\boldsymbol{T}$. However, usually, the number of hidden layer nodes (randomly obtained) is much smaller than the number of training samples. In this case, the matrix is singular and irreversible. So, the process translates to finding the least squares value $\hat{\boldsymbol{\beta}}$ of the output weights $\beta$ :

$$
\|\boldsymbol{H} \hat{\boldsymbol{\beta}}-\boldsymbol{T}\|=\min \|\boldsymbol{H} \boldsymbol{\beta}-\boldsymbol{T}\|
$$

Correspondingly, the generalized inverse matrix can be used to solve the inverse matrix of the singular matrix. If $\boldsymbol{H}$ is non-singular, the generalized inverse matrix is equivalent to $\boldsymbol{H}^{-1}$. If $\boldsymbol{H}^{+}$ is used to represent the generalized inverse matrix of $\boldsymbol{H}$, then $\hat{\boldsymbol{\beta}}$ is calculated as: $\hat{\boldsymbol{\beta}}=\boldsymbol{H}^{+} \boldsymbol{T}$. If $\boldsymbol{H}^{+}$is a Moore-Penrose general inverse of $\boldsymbol{H}$, then the obtained solution $\hat{\boldsymbol{\beta}}$ value is the smallest and unique.

In training progress, the output weight $\beta$ is continuously optimized through iteration, so the learning process of the entire ELM network can be defined as:

$$
\left\|\boldsymbol{H}\left(\hat{a}_{i}, \hat{b}_{i}\right) \hat{\boldsymbol{\beta}}-\boldsymbol{T}\right\|=\min _{a, b, \beta}\left\|\sum_{i=1}^{L} \beta_{i} f\left(a_{i} \boldsymbol{x}+b_{i}\right)-t_{i}\right\|
$$

It should be noted that ELM have absolute advantages in efficiency over other neural networkbased machine learning algorithms. The reasons are: According to the interpolation theorem and the general limit theorem [21] When Neural Network, SLFN(Single-Hidden Layer Feedforward)'s hidden layer mapping function satisfies infinitely differentiability, its learning ability is not related to the selection of parameters such as input weight and threshold (bias), and is only related to the current network structure. Therefore, compared to other methods, the input layer weight a and bias b of ELM can be randomly generated from any continuous probability distribution within any interval of the space under the premise that the activation function is selected as a continuously-dimensionable function. It only requires iterative training of the output layer weights [22].

\subsection{The Process of Network Resilience Prediction by Using ELM}

Step1. Based on the above definition of the prediction model, the network resilience can be predicted by the following method:

Generate input weights and thresholds randomly for $\mathrm{L}$ nodes within the weight range of input weights and thresholds (bias): $\left(a_{i}, b_{i}\right), i=1,2, \ldots, L$, At the same time, a continuously differentiable function is selected as an activation function. In addition to the Sigmoid function, the Radial Basis 
Function (RBF) can be selected as the activation function so that the ELM can avoid overfitting when processing high-dimensional data. The hidden layer hybrid activation function that can be selected is:

$$
\begin{gathered}
\mathrm{A}\left(x, \alpha, \sigma_{i}\right)=\alpha \cdot \omega(x)+(1-\alpha) \sigma_{i} \phi(x) \\
\text { In eq. (15): } \omega(x)=\frac{1}{1+e^{-x}} \\
\phi(x)=\phi\left(x, \mu_{i}, \sigma_{i}\right)=e^{\frac{\left\|x-\mu_{i}\right\|}{\sigma_{i}}}
\end{gathered}
$$

$\alpha$ represents the ratio of the mixture of the two activation functions, $\phi(x)$ is the Gaussian kernel function as the radial basis function, $\boldsymbol{\mu}_{i}$ is the center of the kernel function, and $\sigma_{i}$ is the width of the radial basis function. In this algorithm, in order to facilitate the implementation, the matrix of the hidden layer node $\left(n_{-}\right.$hidden, $h_{-}$features) shape is taken as the value of $\boldsymbol{\mu}_{i}$.

Step 2. Calculate the hidden layer output matrix according to the following formula:

$$
\boldsymbol{H}\left(a_{1}, a_{2}, \ldots, a_{L}, b_{1}, b_{2}, \ldots, b_{L}, x_{1}, x_{2}, \ldots, x_{L}\right)=\left[\begin{array}{ccc}
f\left(a_{1} x_{1}+b_{1}\right) \cdots f\left(a_{L} x_{1}+b_{L}\right) \\
\vdots & \cdots & \vdots \\
f\left(a_{1} x_{L}+b_{1}\right) \cdots f\left(a_{L} x_{L}+b_{L}\right)
\end{array}\right]
$$

Step 3. According to calculating $\hat{\boldsymbol{\beta}}=\boldsymbol{H}^{+} \boldsymbol{T}$, the output weight matrix is obtained. The part that can be improved in this step is: the orthogonal projection method can be used to calculate the generalized inverse matrix $\boldsymbol{H}^{+}$. The method uses the following matrix characteristics: If $\boldsymbol{H}^{T} \boldsymbol{H}$ is a non-singular matrix, then $\boldsymbol{H}^{+}=\left(\boldsymbol{H}^{T} \boldsymbol{H}\right)^{-1} \boldsymbol{H}^{T}$, if $\boldsymbol{H} \boldsymbol{H}^{T}$ is a non-singular matrix, then $\boldsymbol{H}^{+}=\boldsymbol{H}^{T}\left(\boldsymbol{H} \boldsymbol{H}^{T}\right)^{-1}$, according to the principle of ridge regression (which can be achieved by losing some information and reducing certain accuracy to obtain a more realistic and more reliable regression method for the regression coefficient) introduces a positive real number $\frac{1}{\lambda}$ on the diagonal of $\boldsymbol{H}^{T} \boldsymbol{H}$, so $\hat{\boldsymbol{\beta}}=\boldsymbol{H}^{+} \boldsymbol{T}$ can be converted into: $\hat{\boldsymbol{\beta}}=\left\{\begin{array}{l}\left(\frac{1}{\lambda}+\boldsymbol{H}^{T} \boldsymbol{H}\right)^{-1} \boldsymbol{H}^{T} \boldsymbol{T} \\ \boldsymbol{H}^{T}\left(\frac{1}{2}+\boldsymbol{H} \boldsymbol{H}^{T}\right)^{-1} \boldsymbol{T}\end{array}\right.$, corresponding to $\boldsymbol{H}^{T} \boldsymbol{H}$ and $\boldsymbol{H H}^{T}$ are non-singular matrix. Then the calculating process can be converted to a simple liner transformation process.

So far, the prediction model and approach based on ELM was constructed. Essentially, the approach is to find $\hat{\boldsymbol{\beta}}$ according to training a large number of data samples. Once the optimal value $\hat{\boldsymbol{\beta}}$ was obtained, network resilience can be predicted for any input and network designing parameters based on the trained output weight matrix and activation function. A simple case will be used to verify the effectiveness of the method below.

\section{Case Study}

Here we constructed a case to verify the validity of proposed model and method and analyze phyFR and TSFR metrics during network resilience evaluation process.

The case framework is based on a MANET which is applied AODV protocol. With the purpose of trying to closer with the real network usage profile, inspired by the simulation designing of R. Jain, et.al. [14], in this case, we setup the simulation as Table 1 shown: 
Based on AODV routing algorithm, the Time-Spatial network will be generated and formed by valid routes and OD pair nodes as Fig.3 and Fig.4 shown:

Table 1. Simulation Setup

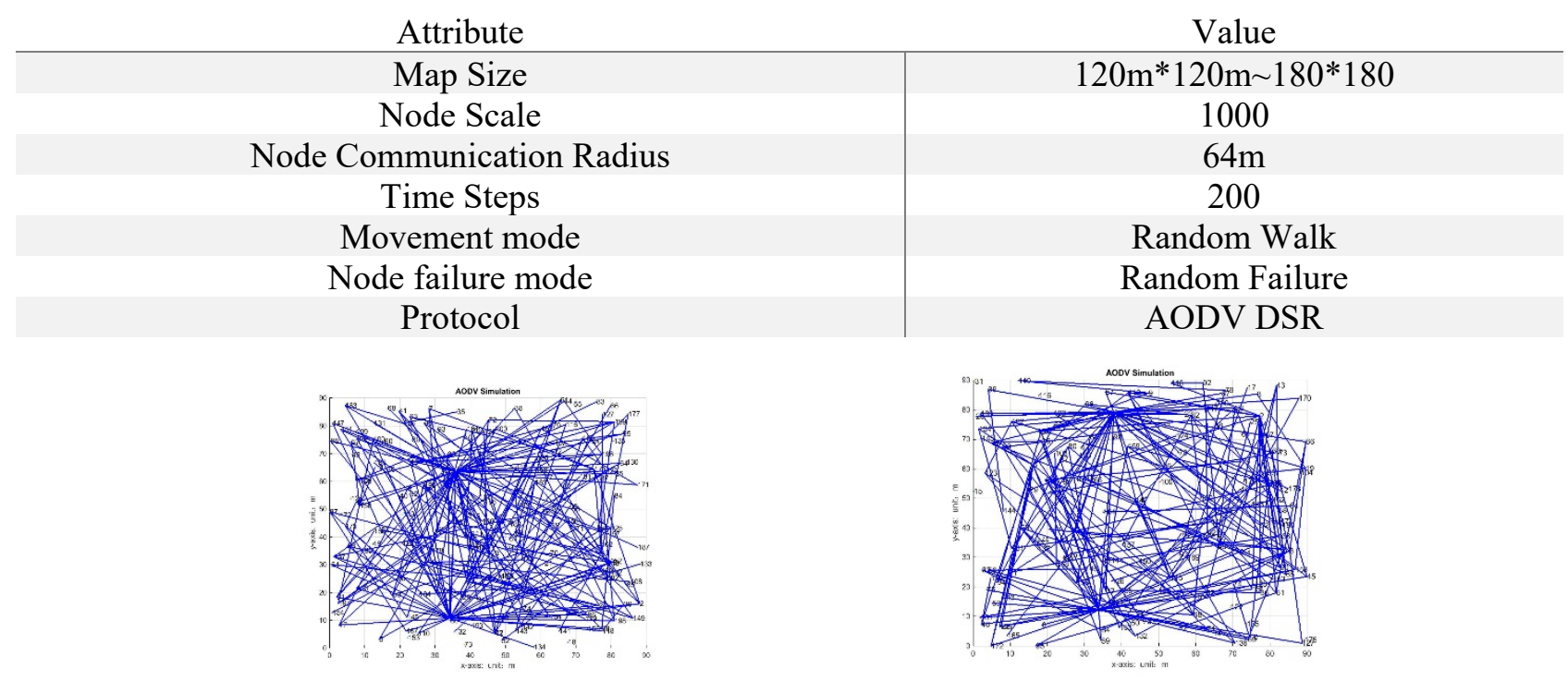

Fig. 3. Available Node Number $=200$

Fig. 4. Available Node Number $=180$

we can obtain the Time-Spatial network topology graph. From observing the generated topology graph at different time points, the topology is varying with time increasing, and the network structure metrics also changes obviously, here we express the Time-Spatial network's structure by the metrics we mentioned in Section 2.1 as Fig.5 shown:

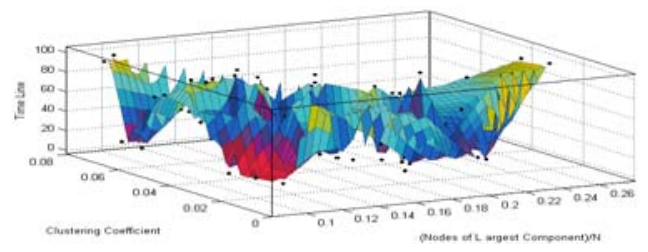

Fig. 5. Clustering Coefficient and Largest Component Varying in Time Dimensions

From the simulation result, we can obtain that liner or non-liner relationship existing between differeent logical fault-tolerance strategies and resilience value of whole network. So, the machine learning approaches can be adopted for predicting the resilience value if the fault tolerance strategies are known.:

Table 2. Simulation Result

\begin{tabular}{|c|c|c|c|}
\hline PDR & LFT & phyFR & Failure nodes num \\
\hline 0.546547 & 0.591592 & 0.783784 & 1 \\
\hline 0.55511 & 0.601202 & 0.793587 & 2 \\
\hline 0.561685 & 0.605817 & 0.807422 & 3 \\
\hline 0.558233 & 0.581325 & 0.791165 & 4 \\
\hline 0.552764 & 0.59598 & 0.78794 & 5 \\
\hline$\ldots$ & $\ldots$ & $\ldots$ & $\ldots$ \\
\hline 0 & 0 & 0 & 1000 \\
\hline
\end{tabular}

After the training data is obtained from simulation, the model can be trained to obtain a good resilience prediction model. The accuracy of the model obtained through training can meet the forecast needs: the accuracy of the training set is $97.07 \%$, and the accuracy of the test set in crossvalidation is $96.43 \%$. Figure 6 is the contrast of predicted value (red fold line) of the entire network 
elasticity through the logical layer fault-tolerance strategy parameters (red dots) and the physical layer fault-tolerance strategy parameters (blue dots), and the real resilience value (golden triangle). From the results, we can see that the proposed method has high accuracy in predicting network elasticity through fault-tolerant strategies.

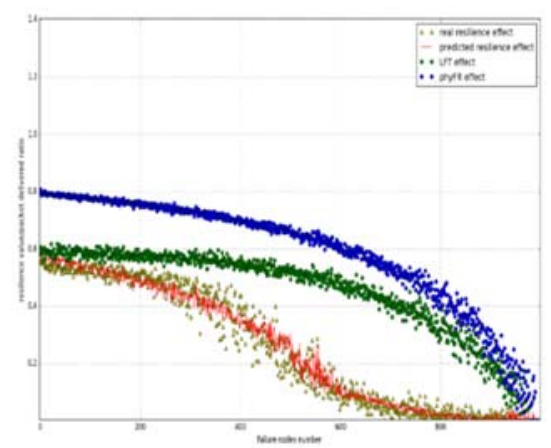

Fig 6. The Result Contrast Between Prediction Value and Labeled Real Value

Based on the prediction model, further, we can try to predict and analyze the resilience level of the entire network based on the single fault tolerance strategy, in order to analyze the effect of the single fault tolerance mechanism on the resilience of the entire network:

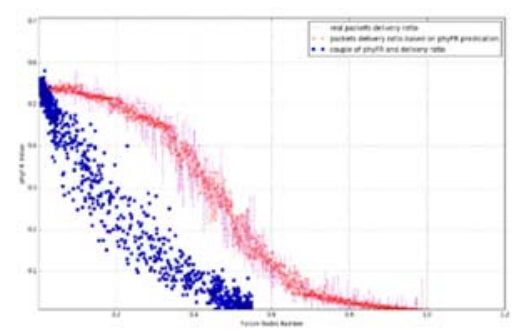

Fig 7. Contrast of Predicting Value According to the Physical Layer Fault Tolerance Strategy Parameters and Real Resilience Value

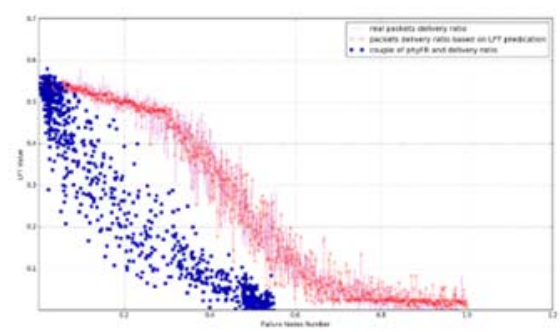

Fig 8. Contrast of Predicting Value According to the Logical Layer Fault Tolerance Strategy Parameters and Real Resilience Value

\section{Conclusion}

In this paper, combining the theory of network elasticity and extreme learning machine, the method of predicting the network elasticity is proposed. Experimental verification shows:

1) A fault-tolerant strategy model based on dynamic characteristics of network space-time can effectively describe and quantify the fault-tolerance capabilities of fault-tolerance strategies.

2) The proposed resilience prediction method based on machine learning can accurately predict network resilience by using quantify parameters of fault-tolerance strategies, and the accuracy rate can reach over $96 \%$. 
3) Through the preliminary analysis of the experimental results, it can be concluded that network resilience is the result of the coupling of multiple fault-tolerance strategies. The mutual influence of multiple fault-tolerance strategies also affects the resilience of the entire network.

\section{References}

[1]. M. Al-Kuwaiti, N. Kyriakopoulos, and S. Hussein, "A comparative analysis of network dependability, fault-tolerance, reliability, security, and survivability," IEEE Communications Surveys Tutorials, vol. 11, no. 2, pp. 106-124, Second 2009.

[2]. I.-R. Chen, A. P. Speer, and M. Eltoweissy, "Adaptive Fault-Tolerant QoS Control Algorithms for Maximizing System Lifetime of Query-Based Wireless Sensor Networks," IEEE Transactions on Dependable and Secure Computing, vol. 8, no. 2, pp. 161-176, Mar. 2011.

[3]. Z. Zheng and M. R. Lyu, "Selecting an Optimal Fault Tolerance Strategy for Reliable ServiceOriented Systems with Local and Global Constraints," IEEE Transactions on Computers, vol. 64, no. 1, pp. 219-232, Jan. 2015.

[4]. A. Zhou, M. Liu, Z. Li, and E. Dutkiewicz, "Cross-layer design with optimal dynamic gateway selection for wireless mesh networks," Computer Communications, vol. 55, pp. 69-79, Jan. 2015.

[5]. J. P. G. Sterbenz et al., "Redundancy, diversity, and connectivity to achieve multilevel network resilience, survivability, and disruption tolerance invited paper," Telecommun Syst, vol. 56, no. 1, pp. 17-31, May 2014.

[6]. S. Hosseini, K. Barker, and J. E. Ramirez-Marquez, "A review of definitions and measures of system resilience,” Reliability Engineering \& System Safety, vol. 145, pp. 47-61, Jan. 2016.

[7]. A. Sydney, C. Scoglio, and D. Gruenbacher, "Optimizing algebraic connectivity by edge rewiring," Applied Mathematics and Computation, vol. 219, no. 10, pp. 5465-5479, Jan. 2013.

[8]. M. J. F. Alenazi and J. P. G. Sterbenz, "Evaluation and improvement of network resilience against attacks using graph spectral metrics," in Resilience Week (RWS), 2015, 2015, pp. 1-6.

[9]. D. Zhang and J. P. G. Sterbenz, "Measuring the resilience of mobile ad hoc networks with human walk patterns," in 2015 7th International Workshop on Reliable Networks Design and Modeling (RNDM), 2015, pp. 161-168.

[10]. J. P. Rohrer, A. Jabbar, and J. P. G. Sterbenz, "Path diversification for future internet endto-end resilience and survivability," Telecommun Syst, vol. 56, no. 1, pp. 49-67, Aug. 2013.

[11]. D. James, J. J. Babu, and S. S. Joseph, "Literature Survey on Establishing Wireless Sensor Networks Using Otway Rees Protocol," International Journal of Emerging Trends in Science and Technology, vol. 2, no. 2, Feb. 2015.

[12]. B. Rong, S. Sun, and M. Kadoch, "Traffic Prediction for Reliable and Resilient Video Communications Over Multi-Location WMNs," J Netw Syst Manage, vol. 24, no. 3, pp. 516533, Jul. 2016.

[13]. J. Gao, B. Barzel, and A.-L. Barabási, "Universal resilience patterns in complex networks," Nature, vol. 530, no. 7590, pp. 307-312, Feb. 2016.

[14]. Y. Chen and H. Yang, "Sparse Modeling and Recursive Prediction of Space -Time Dynamics in Stochastic Sensor Networks," IEEE Transactions on Automation Science and Engineering, vol. 13, no. 1, pp. 215-226, Jan. 2016.

[15]. M. J. F. Alenazi and J. P. G. Sterbenz, "Evaluation and comparison of several graph robustness metrics to improve network resilience," in 2015 7th International Workshop on Reliable Networks Design and Modeling (RNDM), 2015, pp. 7-13. 